\title{
Intervenciones preventivas en la primera infancia en Europa*
}

\author{
BURKHART, G. \\ Observatorio Europeo de la Droga y las toxicomanías (OEDT).
}

Enviar correspondencia a:

Gregor Burkhart. Observatorio Europeo de la Droga y las Toxicomanías (OEDT). Rua de la Cruz de Sta. Apolonia, 23-25. P-1149-045 Lisboa - Portugal Phone direct +351 21811 3022. Fax + 35121813 7943. Http://www.emcdda.org

\section{RESUMEN}

Intervenciones preventivas en la primera infancia constituyen una área poco desarrollada. En pocas regiones Europeas existen programas suficientemente estructurados que puedan darnos una imagen clara y conclusiva sobre las posibilidades y mejores formas de actuación en este campo. Para dar una visión global de la situación Europea se ha acudido a la (a) literatura internacional accesible, (b) información extraída de informes nacionales de los estados miembros al OEDT y (c) descripciones de programas en este ámbito, contenidos en el sistema EDDRA del OEDT. Intervenciones con niños pequeños, sea en los jardines infantiles sea en las familias, son relativamente raras en la UE, a pesar de la importancia que se les atribuye siempre en todas las publicaciones sobre prevención de drogodependencias. En muchos casos, intervenciones que se dirigen a niños pequeños se concentran en hijos de toxicómanos, dejando de lado a las poblaciones de niños con perfil de riesgo muy parecido, probablemente por una fijación en experiencias y perspectivas de servicios de tratamiento, en vez de usar conceptos verdaderamente preventivos de salud pública. En este artículo se identifican y discuten varios conceptos de actuación utilizados en Europa, que pueden servir de base para intercambios e innovaciones que realmente corresponden a las necesidades holísticas de la salud pública.

Palabras clave: revisión, prevención drogas, infancia, Europa

\section{SUMMARY}

Prevention interventions during early childhood are a neglected area in demand reduction, and only in few European regions do programmes exist that are sufficiently structured to offer us a clear and conclusive image regarding possibilities and good practice in this field.

The overview of early childhood interventions in Europe presented in this article draws on a) the available international literature, b) information extracted from national reports submitted by the EU Member States to the EMCDDA and c) programme descriptions from this setting, contained in the EMCDDA's recently established information system, EDDRA. By utilising these diverse information sources, it is possible to estimate the European reality in this scarcely researched field.

Early childhood interventions -be they in kindergartens or families - are relatively rare in Europe, despite the importance continuously attributed to them in publications on drug prevention. Targeting children in their time of infancy, they often tend to concentrate on children of drug users, without considering the larger populations of children with similar risk profiles. This is probably due to a fixation on experiences and perspectives revealed in treatment settings, instead of using truly preventive approaches.

In this article, several approaches used in Europe are presented and discussed. These may serve as starting points for further exchange and innovation corresponding to the holistic needs of public health.

Key words: review, drug prevention, childhood, Europe.

\section{INTRODUCCIÓNY CONTEXTO}

$\mathbf{E}$ I Observatorio Europeo de la Droga' recopila continuamente información sobre las actividades de reducción de la demanda en los Estados miem- bros de la UE. Uno de los subtítulos bajo los cuales se recoge tal información es "intervenciones en la primera infancia". Es este un ámbito escasamente cubierto y al que se presta muy poca atención, aparte de que a menudo su alcance y significado no se comprenden

\footnotetext{
* Reproducción autorizada por la revista Toxicodependências del artículo "Intervençoes na primeira infancia- posibilidades e experiências na Europa"

'El Observatorio Europeo de la droga y las Toxicomanías, con sede en Lisboa, es uno de los organismos descentralizados de la Unión Europea, tiene por objeto proporcionar a los Estados miembros de UE datos objetivos, fiables y comparables sobre las drogas, las toxicomanías y sus consecuencias.
} 
bien, creyéndose muchas veces que se trata exclusivamente de intervenciones dirigidas a hijos de consumidores de drogas.

En 1992, el IFT de Munich, uno de los puntos focales del Observatorio Europeo de las Drogas y Toxicomanías, realizó un meta-análisis ${ }^{1}$ de informes de evaluación de programas de prevención del abuso de sustancias en todo el mundo y concluyó que las medidas preventivas comienzan demasiado tarde: deben comenzar cuando los niños son aún muy jóvenes. El análisis de algunos estudios sugiere que los estilos parentales de educación de los hijos tienen una gran influencia sobre la capacidad de los niños para adquirir las aptitudes que determinan si, según vayan haciéndose mayores, abusarán o no de las drogas. Según el estudio, los niños de 7 a 10 años que después consumen drogas tienden a mostrar características tales como la falta de confianza en sí mismos, incapacidad para llegar a mantener unas relaciones sanas, discapacidad afectiva (falta de sensibilidad). No reciben una atención parental adecuada ni experiencias que favorezcan su desarrollo afectivo; las madres son "frías, críticas, dominantes, y muestran poco interés por las necesidades de sus hijos". De estos resultados se deduce que las medidas preventivas destinadas a mejorar la calidad de la educación parental deben empezar pronto, a más tardar, cuando los niños están entre las edades de cinco y ocho años. Puesto que solamente unos pocos estudios han descubierto hasta ahora estas relaciones causales, cualquier conclusión de carácter general debe formularse con gran precaución.

También a partir de varias otras fuentes, desde que estamos evaluando y midiendo la eficacia de la prevención de la drogadicción en Europa, repetidamente se concluye y se insta a que la prevención primaria de las toxicomanías comience en una fase muy temprana del desarrollo. El hecho, sin embargo, es que la abrumadora mayoría de las intervenciones de prevención en Europa comienza relativamente tarde, y se dirigen a adolescentes en educación secundaria. Por otra parte, la mayoría de las intervenciones a nivel de la familia tienen por objeto interacciones sistémicas en las familias en general, sin responder a fines específicos destinados al desarrollo de los niños de corta edad, ni a mejorar los conocimientos y capacidad de los padres para educar a sus hijos en la primera infancia. Para escribir este artículo se ha hecho uso del sistema de información EDDRA recientemente creado por el Observatorio Europeo de la Droga, así como de datos procedentes de los informes nacionales de los Estados miembros de la UE enviados al Observatorio y otra documentación disponible, para esbozar un primer panorama de las intervenciones en la primera infancia en Europa y sus posibilidades de mejora.

En el ámbito de las intervenciones en la primera infancia pueden distinguirse varias situaciones: inter- venciones dirigidas a las familias (técnicas de educación parental etc....), intervenciones en guarderías y a nivel preescolar, intervenciones dirigidas a las madres después del parto, intervenciones específicas para niños de drogadictos y enfoques comunitarios. La situación en los EE.UU. está bien reflejada en el estudio Hall \& Zigler $^{2}$, que en una encuesta identificaron 41 programas dentro de los currículos escolares para niños a partir de 3 a 5 años en diversos contextos, como guarderías, iglesias y centros comunitarios. La variedad de planteamientos individuales utilizados en estos programas hacía su clasificación muy difícil. No obstante, era posible hacer una distinción aproximada de los diversos planteamientos. El enfoque más generalizado estaba orientado a aumentar la afectividad y desarrollar la autoestima, y el segundo en importancia estaba orientado a la solución de problemas y a la práctica de técnicas de resistencia ("dí simplemente no"). En pocos casos se trataba de educación sanitaria. Los autores han analizado la situación desde un punto de vista crítico: 2/3 de los programas contenían material explícito que incluía muestras de drogas, y la mayor parte de los programas no implicaban en absoluto a los padres. Sólo se hallaban disponibles datos sobre los resultados en el caso de un $27 \%$ de los programas. Se comprobó que ningún programa era capaz de proporcionar una información de tipo longitudinal sobre la eficacia del programa con respecto al objetivo último de frenar el abuso de drogas. La mayoría de los programas no están basados en el conocimiento de los factores de riesgo y resistencia asociados con comportamientos socialmente ineptos, o en lo que se sabe sobre el papel de la familia como agente primario de socialización. El objetivo del estudio no era sin embargo demostrar que los programas incluidos en los planes de enseñanza eran ineficaces, sino que existen dudas sobre el valor a largo plazo de este tipo de enfoques, pues se basan en una consideración de los niños como entes aislados, sin tener suficientemente en cuenta una gran variedad de factores, como el funcionamiento de la familia, el estilo de educación parental, el desarrollo social y cognoscitivo de los niños y los indicadores de aptitud social. También se cuestiona si se retienen suficientemente en la infancia los mensajes específicos de los programas escolares. Como alternativa se proponen intervenciones específicas de apoyo a las familias, que se basan en los resultados prometedores de intervenciones multimodales de amplio espectro en el ámbito de la prevención de la delincuencia juvenil. Cubren una gama más amplia de variables de influencia en los comportamientos desviados que aparecen en etapas posteriores. Los resultados longitudinales de esos planteamientos aportaron beneficios sociales positivos y una buena relación coste-eficacia. Implican a niños en fases muy tempranas o inmediatamente después del nacimiento y muestran buenos resultados con respecto a los indi- 
cadores de aptitud social, éxito escolar y delincuencia juvenil.

Un estudio más reciente ${ }^{3}$ pudo confirmar concretamente - para un período de observación entre el nacimiento y los 10 años- la fuerte relación entre la falta de respuesta emocional de la madre hacia el niño durante la infancia y el desarrollo posterior de trastornos del comportamiento, que por sí mismos son predictores del abuso de drogas en la adolescencia. También existía una fuerte relación al controlar variables sociales, como la situación socioeconómica familiar deprimida, el comportamiento antisocial parental o variables personales como, entre otras, el bajo índice de inteligencia/educación maternal, la maternidad muy temprana, el poco peso al nacer, o los problemas de desarrollo mental. Ni la exposición prenatal a los opiáceos o la marijuana ni la toxicomanía materna contribuían de forma perceptible al modelo logístico de regresión utilizado en el estudio, lo que constituye un hallazgo pertinente a la hora de considerar la importancia del abuso materno de drogas como base lógica para establecer intervenciones específicas de prevención. Los efectos duraderos del comportamiento y sensibilidad maternales en los primeros años pueden explicarse por los cambios neurobiológicos, que están condicionados por las influencias del entorno que actúan en edades tempranas (estrés psicosocial, perturbaciones interactivas) y por la etapa de desarrollo en que se producen.

Este modelo también esta respaldado por los resultados de un estudio retrospectivo de Anda y otros $^{4}$, en el que se confirmó la asociación entre experiencias infantiles adversas (abusos verbales, físicos, sexuales, madre maltratada, enfermedades mentales en el hogar, separación de los padres) y el consumo de tabaco en los adolescentes. El hábito de fumar o el abuso de alcohol/drogas de los padres no intervenía en esta relación. No obstante, este estudio estaba concebido refiriéndose únicamente a los recuerdos de las experiencias infantiles vividas, por lo que no incluye la fase de desarrollo más vulnerable anterior a los 5 años, aunque apoya la necesidad de dirigir los esfuerzos a las interacciones familiares en vez de a la toxicomanía de los padres.

En un artículo sobre los resultados a largo plazo en casos de niños expuestos al abuso de substancias ilícitas en la fase intrauterina ${ }^{5}$ se dice que sus efectos sobre el desarrollo de los niños tienen una duración relativamente breve (hasta los 7 años) y son reversibles, al contrario que ocurre con los efectos del abuso prenatal de alcohol, no presentando ninguna predisposición al abuso de dichas sustancias posteriormente.

En un reciente estudio de evaluación ${ }^{6}$ se presentaron los resultados de un proyecto preescolar para la prevención de la drogadicción y la violencia. El método utilizado consistía en reforzar las cualificaciones de los enseñantes para mejorar el desarrollo emocional y cognoscitivo social infantil en áreas de situación socioeconómica deprimida y de violencia comunitaria. El resultado de proceso y la evaluación final, tanto en términos de medidas cuantitativas como cualitativas, mostraron resultados moderadamente positivos para el profesor y perceptiblemente positivos para los niños. La evaluación estaba planteada de tal modo que permitía realizar revisiones mientras el programa estaba en curso, al descubrir determinados fallos. Como próximo paso en el programa y la investigación está previsto implicar a los padres. El diseño del programa no permitía medir los efectos a largo plazo, pero los resultados a corto plazo confirman el valor de programas no específicos orientados desarrollar la resistencia ante las drogas como parte de un espectro más amplio de intervenciones comunitarias.

\section{Conclusiones y recomendaciones prácticas}

Las conclusiones de los estudios anteriormente mencionados dieron lugar a recomendaciones de intervenciones en la primera infancia en un amplio abanico de situaciones, implicando a la familia, la escuela y a instituciones de apoyo, resultando en experiencias positivas en el hogar, la escuela, etc. Las intervenciones capaces de modificar el funcionamiento y las interacciones familiares prometen mejores resultados a largo plazo y son más receptivas ante las distintas características culturales que las intervenciones de amplio espectro incluidas en los planes de enseñanza. Es igualmente importante que las intervenciones traten de integrarse ecológicamente en el mundo infantil (y familiar) de nociones y normas, sin concentrarse exclusivamente en la información o formación con respecto al abuso de substancias.

\section{NIVEL DE ACTIVIDAD EN MATERIA DE INTERVEN- CIONES EN LA PRIMERA INFANCIA EN LOS ESTA- DOS MIEMBROS DE LA UE}

La siguiente información procede de los informes nacionales anuales que los Estados miembros de la UE remiten al Observatorio Europeo de las Drogas y Toxicomanías para proporcionar un panorama de la situación real en cuanto a las actividades en curso en la Unión Europea. La información contenida en dichos informes es a menudo genérica, de modo que lo que se expone a continuación es una selección de las partes más concretas de los informes nacionales.

Austria informa que los planteamientos "orientados hacia la información sobre las drogas" han sido sustituidos por el concepto de "prevención de la adicción", centrándose en la promoción de la salud y la educación así como en el desarrollo y refuerzo de la 
personalidad. En este contexto, se descubrió que los niños debían ser un grupo destinatario de la prevención primaria por lo que las actividades se destinan también a los niños en edad preescolar. El taller SUPRO para la prevención de la adición en Vorarlberg puso en marcha la campaña de información "proporcionar a nuestros hijos la capacidad para poder decidir", cuyo principal objetivo era hacer a la población de Vorarlberg consciente de la importancia de la prevención temprana de la adición. Tomando como base los resultados de esta campaña de prevención se empezará otra campaña para continuar el proceso de sensibilización y para aplicar medidas concretas. El objetivo global del proyecto es reforzar las competencias para la vida de niños y jóvenes. Los aspectos relativos a la igualdad entre hombres y mujeres también se están teniendo cada vez más en cuenta en las actividades destinadas a prevenir la adicción". Alemania hace referencia a un informe especializado de Kämmerer que evalúa el material existente para la prevención de la adición en la edad preescolar y proporciona una descripción detallada de este dominio. "Como ejemplo reciente de actividades en este ámbito se presenta el proyecto conjunto de las unidades de prevención de droga en Hamburgo titulado 'infancia sólida - vida sólida'. Está dirigido principalmente a los padres y a los educadores de sexo masculino o femenino en las guarderías. Los módulos del proyecto consistían en encuentros de información, formación profesional avanzada, ayuda organizativa y cuidado intensivo en las tareas prácticas". Según las experiencias griegas, "son mayoritariamente las madres quienes asisten a los programas de intervención familiar. La limitada implicación de los padres en los programas de prevención parece provenir no sólo de impedimentos laborales, sino también de que en la sociedad griega se considera que la educación y el bienestar de niños son responsabilidad de la mujer. Un pequeño número de centros de prevención está haciendo esfuerzos sistemáticos para resolver esta dificultad tratando de hacer que los padres sean conscientes de que el papel del padre en la educación infantil es primordial" El informe griego expone claramente que "faltan programas para padres de niños en escuelas primarias (con edades de 6 a12 años) o niños más pequeños". También en el informe español se admite que las "intervenciones dirigidas a niños de menos de cuatro años de edad no son muy frecuentes tanto dentro como fuera del entorno escolar". En cualquier caso, las situaciones especialmente difíciles por razones de índole familiar o social, que afectan a los niños pequeños, son tratadas por servicios especializados. Existen, sin embargo, programas que trabajan con hijos de alcohólicos.

En otros países (Reino Unido, Bélgica, Suecia y Dinamarca), predominan los enfoques específicamente destinados a hijos de consumidores de drogas, como en Dinamarca, donde "se ha creado un proyecto familiar con el fin de asegurar a los niños en familias de toxicómanos una educación sana con los menores cambios posibles del entorno en que crecen y de las personas que se ocupan de ellos. Este proyecto surgió porque a menudo los niños de estas familias eran niños que iban "de acá para allá" acompañando a sus padres a los diversos centros de tratamiento para adultos sin que se prestase ninguna atención a su propia situación y necesidades infantiles. Los centros de observación y tratamiento en algunos lugares observan y tratan a niños de 0 a 7 años con padres toxicómanos."

Sin embargo, en el Reino Unido existen medidas específicas para la infancia pero no limitadas a los hijos de drogadictos. Un ejemplo es el "conjunto integral" de servicios de atención a los hijos de padres noveles que ha puesto en marcha el Gobierno con el fin de reducir los costes sociales de una deficiente educación parental. El programa "SURE START" (BUEN COMIENZO) reunirá a los servicios de educación, sanidad y servicios sociales para niños pequeños, financiados con $£ 540$ millones puestos a disposición en las previsiones presupuestarias de este año. Los problemas de la droga se abordarán a través de este programa. Bajo este sistema, todos los padres noveles serán visitados por un representante del programa antes de que el bebé cumpla los tres meses, para ofrecer asesoramiento sobre atención sanitaria, alimentación, servicios locales de cuidado de niños, centros de juego y apoyo adicional a niños y padres con necesidades especiales físicas o de comportamiento debidas al uso problemático de drogas. Estos visitadores de SURE START, que tendrán su base en escuelas o centros de sanidad locales, complementarán los servicios que ya ofrecen los visitadores sanitarios. El Gobierno pretende establecer inicialmente 250 centros SURE START en un plazo de tres años. De manera más general, también los Países Bajos y Dinamarca describen acciones similares. En los Países Bajos, unas 14 organizaciones en el ámbito de la drogadicción proporcionan educación y apoyo parentales. Estas intervenciones de tipo preventivo se encuentran en período experimental y todavía no es posible evaluar su eficacia. El objetivo de un trabajo de investigación realizado sobre este tema es evaluar los factores importantes de riesgo y los factores protectores así como evaluar las posibilidades de llevar a cabo estudios sobre el efecto de estas intervenciones de formación y apoyo de los padres ${ }^{8}$. La mayoría de los 275 municipios en Dinamarca han formalizado la cooperación entre la escuela, los servicios sociales y la policía, Ilamada cooperación SSP. El objetivo de esta cooperación intersectorial es captar señales, también para combatir las malas condiciones de vida generales y específicas de niños y jóvenes, proponer y poner en marcha 
actividades, y actuar con carácter preventivo en relación con la drogadicción y la delincuencia. SSP puede, por ejemplo, participar en los trabajos de las escuelas y de los clubes de la juventud con información sobre las drogas, así como poner en marcha actividades a nivel de la calle y crear proyectos especiales para captar a los jóvenes sujetos a los riesgos de la delincuencia y del abuso de drogas.

Resumiendo, es evidente que en todos los países el objetivo general es promover los vínculos parentales, fomentando las competencias y las actitudes afectivas. Los enfoques se basan en la idea de que una fuerte integración madre-hijo, la capacidad de posponer el logro de la gratificación de deseos, de aceptar frustraciones y de evitar el aburrimiento son factores protectores conocidos contra los comportamientos adictivos.

Más allá de estas declaraciones genéricas, parece existir una tendencia en países como Alemania, Austria, Italia y España en centrarse más intensamente en las intervenciones tempranas de prevención con niños pequeños, mientras que en Dinamarca, Francia o Bélgica la atención se dedica más a los problemas de tratamiento y a los hijos de drogadictos.

Únicamente el ejemplo del Reino Unido se encuentra más en la línea de las propuestas de Hall \& Zigler $^{2}$ de realizar intervenciones especialmente dirigidas a la población infantil de riesgo, aunque no específicamente en relación con las drogas, en vez de realizar programas de carácter amplio con objetivos genéricos incluyéndolos en los planes de enseñanza. Para concluir, el panorama europeo parece ser similar al descrito en los EE.UU. por los autores antes citados, pero aquí, en Europa, al parecer se considera que el papel de la familia en las intervenciones tempranas es mucho más importante.

\section{INTERVENCIONES CONCRETAS Y SUS CONTEX- TOS RESPECTIVOS EN EUROPA}

Durante los últimos 3 años, el Observatorio Europeo de la Droga ha comenzado a elaborar un sistema de información directo y continuo sobre actividades relacionadas con la reducción de la demanda de drogas, denominado EDDRA ("E xchange on D rug D emand $\mathbf{R}$ eduction $\mathbf{A}$ action" - Sistema para el intercambio de información sobre acciones destinadas a reducir de la demanda de drogas) que es totalmente accesible a todos los usuarios de Internet en la página del Observatorio en http://www.emcdda.org. Con un enfoque más específico, basado en los programas, contiene una selección de los programas de los Estados miembros de la UE que incluyen un protocolo mínimo de evaluación, proporcionando así una información coherente sobre los objetivos, planteamiento y análisis razonado de los programas, así como infor- mación sobre los resultados de su evaluación que debe ser comprensible y plausible. Los siguientes ejemplos proceden de programas de intervención en la infancia temprana incluidos en EDDRA que han proporcionado algún tipo de resultados a partir de unos

\section{A. INTERVENCIONES EN LA FAMILIA}

Humlan (el abejorro) es un programa sueco destinado a proporcionar un apoyo temprano a los niños y adolescentes con un comportamiento problemático y dificultades de ajuste. Su finalidad es permitir que actúen de forma más adecuada en la escuela, en su tiempo de ocio y en casa, y que encuentren su propio papel en diversas situaciones, así como apoyar a los padres en el ejercicio de su función. El método se basa en contactos estrechos y diarios que se centran en las soluciones, no en los problemas, y en trabajar paralelamente con el niño y con su familia. El modelo se basa en la teoría de que la familia es un sistema operativo en el sentido de que, si un miembro de la familia cambia, ello afecta a todos los demás. La sociedad y la familia se contemplan bajo este mismo prisma de interrelación. El programa alega que el impedir que uno de esos niños pase un año en una institución en el futuro cubre los costes anuales totales del proyecto.

La evaluación muestra tres variables que han contribuido al éxito de Humlan:

- Buena cooperación entre las distintas autoridades (escuela y servicios sociales)

- Actitud positiva en cuanto al tiempo: tiempo es lo que esos niños necesitan y a cada niño se le dedica el tiempo que necesite.

- La actitud positiva del líder, que es afectuosa y de apoyo.

Para realizar la evaluación, se entrevistó a los padres sobre parámetros tales como las relaciones familiares, la independencia y la concentración; en las respuestas se mencionaba "más tolerante con sus amigos, más razonable, va a la escuela, relación estrecha, comunicación más fácil".

El Centro Federal Alemán de Educación (BZGA) ha desarrollado programas para los padres con una cobertura geográfica amplia y con el objetivo global de proporcionar formación a las familias para prevenir el uso indebido de drogas. El punto de partida es que el trabajo preventivo primario debe centrarse en el período anterior al del comienzo de la adicción y abuso y que las medidas preventivas en materia de drogas son efectivas si se relacionan con experiencias subjetivas previas. Los niños necesitan amor, aceptación y confianza para desarrollar una personalidad sana. Como a menudo es difícil para los padres de niños pequeños proporcionarles las condiciones de vida necesarias 
para lograr este objetivo, el BZGA elaboró un sistema de instrucciones para la formación de familias en todas las áreas (rurales, urbanas, y metropolitanas). El objetivo del programa es apoyar y sensibilizar a los padres (dando ejemplos de su propio comportamiento en cuanto al uso de sustancias) sobre su propio papel para preparar a sus hijos que se abstengan del abuso de sustancias. El programa está asociado a una campaña a nivel nacional en los medios de comunicación: "Haga fuertes a los niños."

Los resultados de las entrevistas de seguimiento (6 meses más tarde) mostraron que los participantes continuaban aplicando el contenido de los seminarios en su vida diaria, en especial en cuanto a la motivación para reforzar la autoestima infantil en las actividades cotidianas. Como consecuencia del proyecto, los participantes en los seminarios son capaces de desempeñar el papel de multiplicadores en el ámbito de la formación familiar sobre prevención en materia de drogas. Sin embargo, este planteamiento del programa se enfrenta al problema de los efectos de la selección, que se dan con frecuencia en los proyectos de gran envergadura. Con este tipo de planteamiento es más probable llegar a familias que ya disponen de recursos psicosociales satisfactorios que a familias de riesgo que a menudo no acuden a este tipo de formación de adultos/familias, por varias razones: falta de información, falta de tiempo, falta de interés, y el hecho de no encontrarse tales iniciativas entre sus prioridades.

En Hesse, un concepto innovador piloto para la prevención de la adicción se compone de tres módulos vinculados al proyecto (información, estímulo, psicomoción). Mientras que el módulo de información proporciona a los padres y a educadores la ya bien conocida información sobre la prevención de la drogadicción, la llamada formación de estímulo para los educadores (supervisión) y un programa psicomotor conceptual motivo para los niños en la guardería son conceptos nuevos en la prevención de la adicción. Los elementos de la parte psicomotriz ofrecen a los niños incentivos y experiencias gratuitas, alegres y lúdicas necesarias para madurar. Las experiencias de tipo corporal, material y social se consideran pertinentes. El proyecto se sigue y evalúa científicamente.

\section{B. PROGRAMAS DE GUARDERÍA}

La guardería sin juguetes es un enfoque desarrollado en Alemania y evaluado en Austria. En este programa, todos los juguetes se van retirando sucesivamente de la sala de la guardería dentro del contexto de un cuento de hadas narrado a los niños, en el que se les anuncia que los juguetes necesitan vacaciones de verano. Durante este tiempo los cuidadores de la guardería no sugieren juegos ni otro tipo de activida- des para que pasen el día los niños. Seguidamente se anima a los niños a desarrollar otras formas de juego o a construir nuevos juguetes por sí mismos. Se celebran varias reuniones con los padres para proporcionarles asesoramiento e información y para integrarles en el proyecto. Los cuidadores de la guardería observaron cómo los niños se comportaban durante el período de tiempo en que no tuvieron juguetes $y$ documentaron sus observaciones en inventarios analíticos. Se realizaron evaluaciones continuas que mostraron un aumento significativo de la interacción social, creatividad, expresión (verbal y no verbal) de necesidades y emociones, capacidad para resolver conflictos, flexibilidad en los papeles de los sexos, empatía y confianza en sí mismos con respecto a un grupo de control. Además de estos parámetros, se observó también un aumento de la paciencia y de la tolerancia ante la frustración. Los niños que participaron en este proyecto jugaron bastantes más juegos de movimiento y realizaron importantes avances en su desarrollo de motor. La aceptación por parte de los padres fue satisfactoria.

Ahora el concepto teórico del proyecto de guarderías sin juguetes ya se ha puesto en práctica en cinco guarderías en Burgenland, Austria, como proyecto modelo.

\section{INTERVENCIONES PARA JÓVENES MADRES}

En Alemania se inició recientemente un proyecto experimental en la región de Berlín. Sus objetivos se derivan del resultado de un estudio danés en el que se concluía que los hijos de madres que fuman era más probable que también se hicieran fumadores en la adolescencia. Se contactó a las jóvenes madres fumadoras en las clínicas de obstetricia de Berlín y se les invitó a participar en un programa que incluía al mismo tiempo la prevención de la recaída en el hábito de fumar y los cuidados y educación infantiles. El diseño de esta intervención (con un grupo de control) prevé sesiones de refuerzo cada medio año y una evaluación anual de seguimiento del hábito de fumar en la madre y de la relación madre-hijo. El programa está en curso y todavía no se dispone de resultados de evaluación. No se encuentra aún accesible en EDDRA debido a su reciente inicio.

\section{INTERVENCIONES PARA PADRES DROGADICTOS}

Un programa belga ("educación para la paternidad") aspira a concienciar a los profesionales de la infancia sobre los problemas que surgen cuando retiran a los niños del cuidado de sus padres drogadictos. Este problema parece estar decreciendo puesto que en el 
marco del programa se están negociando contratos detallados con los servicios de asistencia a la juventud que permiten que cada vez más niños permanezcan bajo la custodia de sus madres. El objetivo del programa es mejorar la calidad de la relación entre los padres y sus hijos, así como mejorar la imagen social que los padres tienen de sí mismos, ya que la atención se concentra más en su paternidad que en su drogadicción. Para llegar al grupo destinatario de este proyecto para padres, se visita a la madre y a su bebé en la maternidad y se sensibiliza al personal de enfermería neonatal sobre la problemática en cuestión. La evaluación, según datos de los servicios de ayuda a la juventud y hospitales de obstetricia, mostró un descenso en el número de bebés y niños que se retiraban de la custodia familiar.

\section{E. INTERVENCIONES EN LA ESCUELA PRIMARIA}

Considerando que la escuela es el lugar adecuado para enseñar a niños la educación sanitaria, la comunidad francófona belga puso en marcha un programa dentro del ámbito de la estrategia general de fomento de la salud. El programa responde a la petición de los profesores de conseguir útiles que puedan ayudarles en las aulas a hablar adecuadamente sobre la prevención de las adicciones.

Los objetivos eran fomentar una forma consciente de vida en los niños enseñándoles cómo gestionar su tiempo, su futuro y sus propios recursos. Considerados como grupo, se proponía enseñar a los niños a tener un espíritu crítico para resistir la presión social de sus pares. El $80 \%$ de los usuarios se mostró totalmente satisfecho con el conjunto de útiles de ayuda que les fue proporcionado.

\section{F. OTROS PLANTEAMIENTOS}

Otros planteamientos, que podrían incluirse en el grupo de las intervenciones infantiles tempranas, utilizan técnicas de trabajo de calle o de captación. Como ejemplo podemos considerar el juego denominado "Aventura en la ciudad", un juego de "rol", aplicado en distintos contextos geográficos en siete ciudades portuguesas. Seleccionando a los participantes según criterios tales como fracaso escolar o abandono de los estudios, así como escasa aptitud social y baja autoestima, el proyecto se acerca a los chavales en un contexto lúdico para fomentar formas de vida sanas, por ejemplo: gestión de las emociones, toma de decisiones, gestión de los recursos comunitarios, resistencia a la presión de otros chicos de su edad, relaciones interpersonales, etc.. La utilización del juego como estrategia se basó en la necesidad de utilizar un nivel de comunicación adaptado al grupo des- tinatario, y en la rentabilización de los aspectos formativos de las actividades lúdicas. La intervención se basa en experiencias de grupo donde situaciones imaginarias se afrontan en el contexto de un que juego de "rol". Como en un juego de búsqueda del tesoro, los jugadores buscan pistas para encontrar algo o a alguien perdido. La historia cambia según la edad de los jugadores. Los materiales diseñados para el proyecto los realizan los profesores con el apoyo de recursos comunitarios, de padres, chavales de más edad, etc.. Se forma a los profesores y a los asistentes sociales para enfocar la prevención de la drogadicción utilizando este tipo de metodología. La evaluación de los niños, con respecto al lugar de control no reveló cambios significativos, lo que prueba que es necesario realizar un nuevo diseño de la evaluación. Desde otra óptica, hay varios indicadores positivos: el entusiasmo de niños y profesores, la aceptación de los padres a participar, la creciente demanda de que este proyecto se aplique en nuevas áreas geográficas y en nuevos contextos.

\section{G. CONCLUSIONES}

EDDRA debe en principio contener información resumida y concisa sobre el contenido de los programas, por lo que evidentemente no responde a necesidades de descripción detallada y profunda de programas. Así pues, determinadas cuestiones relativas al contenido exacto de las actividades y su valoración por separado no encontrarán respuesta aquí. En este contexto, conviene aclarar que la selección de programas que se presenta en el presente artículo no pretende cubrir de forma exhaustiva todo el ámbito o número de iniciativas existentes en Europa destinadas a la primera infancia. Como ya se ha dicho, EDDRA sólo incluye los programas que proporcionan información con determinado formato normalizado y que responden a unos mínimos compromisos de evaluación que permitan comparar la información. Ello no presupone un testimonio del grado de la eficacia de los programas incluidos en EDDRA ni sobre los programas que no se han incluido en este sistema. En un futuro próximo podrá obtenerse en EDDRA información sobre el seguimiento de los programas anteriormente mencionados, ya que este sistema de información controla y pone al día los programas en curso a intervalos regulares preestablecidos.

En resumen, en Europa pare haber un abanico de enfoques y escenarios diversos de intervención desde la infancia para prevenir el abuso de drogas; una situación bastante diferente de la que existe en los EE.UU..

1. Las intervenciones a menudo tienen por destinatarios a madres y padres en tanto que grupos estratégicos, con frecuencia a partir del nacimiento 
de un hijo. Las acciones se centran en la capacidad educadora de los padres y en el modo de educar de forma que se refuercen los factores de protección en sus hijos. El problema de cómo llegar al grupo destinatario se aborda, bien mediante anuncios en los medios de comunicación de masas o poniéndose en contacto con las madres en los hospitales de obstetricia.

En la práctica sigue existiendo el problema de no llegar a grupos o familias de alto riesgo con unos programas cuya concepción peca de ser excesivamente genérica. Tampoco está todavía claro - debido a los límites de la información incluida en EDDRA- hasta qué punto estos programas abordan realmente los factores específicos que son pertinentes para la predicción de un posterior comportamiento problemático (como la sensibilidad materna, el contacto físico, etc....) y si los abordan en el momento adecuado de las etapas de desarrollo de los niños. El término "capacidad educadora de los padres", utilizado para describir el contenido de los programas, es demasiado genérico y puede, por ejemplo, referirse únicamente a las competencias parentales de educación social y cognoscitiva de valores etc. por parte de los niños, en vez de a las interacciones parentales de tipo más bien emocional con los niños y bebés, a pesar de que éstas sean neurobiológicamente más pertinentes. En la presente descripción de la situación en Europa no se han hallado intervenciones específicas destinadas a formar a las familias de riesgo en una serie de capacidades parentales específicas, según recomiendan Hall \& Zigler ${ }^{2}$. Es posible que exista este tipo de intervenciones a pequeña escala o con carácter local, por lo que no han llegado al conocimiento de los proveedores de información suprarregionales, lo que refleja una de las limitaciones de EDDRA anteriormente descritas.

A esta categoría pertenecen también las intervenciones específicas destinadas a las madres drogadictas que están criando a sus hijos. Las políticas en la UE en cuanto a apartar a los hijos de las madres drogodependientes varían muchísimo en la práctica. En Europa Central y del Norte parece haber una tendencia cada vez mayor a permitir que las madres drogodependientes permanezcan con sus hijos, o al menos a estabilizar la relación entre hijos y padres. Los países europeos más meridionales, como Grecia, España, Italia y Portugal, tienden a confiar más en las estructuras tradicionales de familias extendidas para que se ocupen adecuadamente del cuidado de los hijos.

2. Las intervenciones pueden tener lugar en la guardería, con el objetivo directo de establecer modelos y capacidades de interacción social en los niños, en un contexto externo al de la familia y en el que los niños pasan un tiempo considerable.

3. Las intervenciones en escuelas primarias a menudo se basan en una estrategia de fomento de la salud e higiene y en las competencias sociales. Estas intervenciones no parecen estar muy estructuradas ni evaluadas. También parecen concentrarse más en la educación sanitaria que en la capacitación social o emocional. Las orientaciones o experiencias de evaluación para crear tales programas, como en el caso del estudio presentado anteriormente6 al parecer no se hallan disponibles en Europa.

4. Es posible aplicar metodologías de captación, para tratar de llegar a niños socialmente marginados con el fin de integrarles mejor en su comunidad y trabajar con ellos en un contexto informal, fuera de la escuela. Este tipo de programa podría ser útil para entrar en contacto con las familias de riesgo a las que no se llega con los programas normales para padres o familias, y su proyección comunitaria es prometedora.

\section{IMPLICACIONES DETIPO PRÁCTICO}

Es difícil obtener un panorama de las técnicas de punta en intervenciones infantiles para la prevención de la drogadicción en Europa. En los informes nacionales enviados al Observatorio Europeo de las Droga y Toxicomanías la información sobre este tema es escasa a pesar de su importancia. Los programas que se exponen en el presente artículo proceden del sistema de información EDDRA del Observatorio que se encuentra en Internet y que contiene una amplia gama de programas destinados a reducir la demanda de la Unión Europea. Habida cuenta de que este sistema es accesible sin restricciones, constituye un útil válido no sólo para la recopilación y difusión de información y para estar al corriente de las nuevas tendencias, sino también para intercambiar experiencias y conectar con nuevas redes sobre cuestiones específicas en toda Europa. Sin embargo, resulta evidente con sólo esta descripción resumida que es preciso aumentar el número de programas incluidos en EDDRA y mejorar la calidad de la información proporcionada para representar mejor la situación real en Europa de las actividades orientadas a la reducción de la demanda. Para lograr este objetivo es necesario mejorar la capacidad de evaluación de los diseñadores europeos de programas en general. Si se realiza un análisis crítico de los programas, la mayoría de los ejemplos que aquí se mencionan no proporcionan una información profundizada ni una evaluación exhaustiva de los resultados. Para ayudar a los profesionales a elaborar conclusiones pertinentes e interesantes a partir de la información que intrínsecamente contienen sus programas, el Observatorio ha preparado unas guías de evaluación de la prevención. Éstas se hallan disponibles en las 11 lenguas de la Unión Europea y se han distribuido hasta ahora a más de 1000 
programas en Europa. Están destinadas preferentemente a los profesionales de los programas (en vez de a los expertos) para permitirles evaluar su propio programa a partir de un concepto más amplio de la evaluación. Consideramos estas directrices como una herramienta útil para elaborar información tangible y comprensible sobre el contenido de los programas que pueda ser utilizada por otros planificadores de programas y proporcione orientaciones a los responsables de la toma de decisiones en materia de prevención.

Al hablar de los criterios de evaluación y calidad en los programas para los niños pequeños, conviene hacer algunas consideraciones. No cabe duda de que en las edades muy tempranas ninguna intervención puede dirigirse específicamente a la prevención de las toxicomanías en etapas posteriores. Por consiguiente, la evaluación de las intervenciones en la primera temprana debe concentrarse en las variables intermedias conocidas de las que tenemos noticia por las investigaciones sobre los factores de riesgo y los factores protectores, tales como la autoestima, la capacidad emocional y para relacionarse, la tolerancia ante la frustración. La relación entre esas variables y el abuso de sustancias es lo suficientemente conocida a través de la documentación internacional como para que las intervenciones durante la primera infancia no necesiten probar en su plan de evaluación que alcanzan el objetivo final de reducir el abuso de sustancias para poder definirse como intervenciones para prevenir la drogadicción. Desde un punto de vista más científico, los recientes resultados de estudios sobre factores de riesgo mencionados al principio se han aplicado sólo de forma insuficiente en las intervenciones de prevención. La mayor parte de los trabajos de investigación sobre prevención no se dedican al área de las intervenciones, donde existe una gran necesidad de actuación. Existe aún una discrepancia entre, por una parte, el conocimiento y potencial derivados de la investigación sobre la resistencia de los niños en situaciones de riesgo y, por otra parte, la realidad de las intervenciones en Europa.

En la presente exposición, las intervenciones dirigidas a hijos de drogadictos se mencionan intencionalmente menos, a pesar de que ocupan más el centro de atención del público y de los profesionales del ámbito de la droga. Dichas intervenciones son importantes y se ocupan de un aspecto pertinente del espectro más amplio de cuidados destinados a los drogadictos y su papel de padres. Sin embargo, se concentran muy específicamente en los aspectos relativos a las drogas en sí mismas y se centran en los drogadictos y su situación y modo de vida; pero desde la perspectiva de la salud pública los hijos de los consumidores de drogas ilícitas constituyen un grupo relativamente pequeño dentro de población de riesgo durante la infancia temprana con respecto a los gran- des grupos de niños en situación de riesgo a causa de su exclusión social y/o por criarse en familias necesitadas. A estos conceptos los denomino "enfoques patologizadores", porque parten de un enfoque muy específico en la patología (social y psíquica) de los drogadictos y extrapolan los modelos explicativos respectivos a otros grupos de la población sin tomar en consideración las necesidades muy distintas de prevención de grupos mayores de riesgo que no pasan por los centros de tratamiento de la drogadicción porque sus padres no consumen drogas ilegales Como demuestra el estudio de Wakschlag y $\mathrm{Hans}^{3}$, es la respuesta emocional materna lo que supone un factor de riesgo pertinente con respecto al comportamiento problemático posterior, independientemente del hecho de que las madres consuman drogas o no. La información procedente de los Estados miembros apoya esta conclusión.

Al analizar la situación de la investigación europea con respecto a este punto, a primera vista el panorama se presenta contradictorio. Un estudio irlandés sobre los efectos sociales y psicológicos sobre los niños pequeños del uso parental de la heroína (niño$\mathrm{N}=10$ ) se deduce que solamente algunos niños mostraban signos de problemas sociales-emocionales, aunque, por otra parte, la mayoría experimentaban dificultades en la escuela. Los asistentes sociales pertinentes entrevistados para el estudio estaban particularmente preocupados por la cuestionable calidad y consistencia de los cuidados prodigados por los padres drogadictos, el riesgo de negligencia física y la posibilidad de que los niños fueran testigos del uso de drogas. Estas preocupaciones se expresaban en un editorial reciente donde se señala que no puede asumirse que la drogodependencia conlleve automáticamente una disminución de la capacidad de ejercer adecuadamente las funciones parentales o que el desarrollo de niños resulte necesariamente afectado de forma desfavorable por la drogadicción parental. Otro estudio irlandés también identificó una serie de variables que paliaban los efectos de la drogadicción parental en los niños, entre otros: las disposiciones adoptadas para cuidar a los niños, el apoyo social del que disponen los padres, en qué medida uno o ambos padres están en tratamiento, si el progenitor drogadicto está o no en tratamiento, el historial de consumo de drogas y tratamientos, la edad del niño cuando los padres comenzaron a utilizar heroína y si el uso de esta droga era caótico.

Una asociación austríaca llevó a cabo una pequeña encuesta ${ }^{12}$ en la que se investigó a 23 madres drogadictas y su relación con sus hijos. Los niños examinados mostraron una amplia gama de anormalidades de comportamiento; por ejemplo, en el ámbito de la interacción social, incapacidad para ajustarse a las normas sociales, problemas sociales en sus relaciones con otros niños y, en algunos casos, una fuerte agresivi- 
dad. En el dominio de las emociones, se observaron intensos grados de ansiedad, disforias, trastornos del lenguaje y/o desarrollo tardío del lenguaje, trastornos psicosomáticos y baja tolerancia ante la frustración. Casi todos los niños examinados también mojaban la cama. Las madres entrevistadas eran emocionalmente regresivas y a menudo mostraban fuertes celos de sus hijos u odio hacia ellos, o consideraban a sus hijos como substitutos de un compañero sentimental. Un estudio danés ${ }^{13}$ sobre 89 niños de edades entre 1 y 10 años mostraba que los niños habían tenido unos comienzos difíciles desde el momento en que nacieron. Casi todos los niños habían sido retirados del cuidado de su madre que aún era drogadicta, sin haberse adoptado ningún tipo de medidas de asistencia social o de tratamiento. En el momento en que se realizó el control para este estudio, solamente un $25 \%$ de los niños se había desarrollado normalmente. Un $21 \%$ mostraba retrasos en su desarrollo psicomotor, de los cuales un $10 \%$ de forma grave. Un 54\% mostraba trastornos de comportamiento y mentales y dos niños habían muerto siendo bebés. Pero estos resultados se debían a que el $56 \%$ de los niños habían sido retirados del hogar y el $80 \%$ del total de niños había sido retirado del hogar en una o varia ocasiones. Los primeros entornos en que se habían desarrollado los niños se caracterizaban por numerosos cambios de lugar y del personal que se ocupaba de ellos y por la inestabilidad, inseguridad, la falta de conexión y de contacto con los servicios de ayuda.

Un estudio francés ${ }^{14}$ sobre 85 hijos de drogadictos que estaban recibiendo tratamientos de substitución mostraba que el $51 \%$ de los padres tenía dificultades para criar a sus hijos y que un 38\% de ellos tenía problemas de comportamiento y manifestaba síntomas de ansiedad y de depresión. Los niños de menos de ocho años mostraban principalmente características de hiperactividad, manifestaciones de oposición, síntomas de ansiedad y de depresión. Los de más de ocho años presentaban principalmente problemas de comportamiento. Un documento portugués ${ }^{15}$ sobre un proyecto experimental de apoyo a los hijos de drogadictos en un centro público de tratamiento especializado confirmaba que con frecuencia los hijos de los clientes del centro mostraban "parcelas problemáticas en su desarrollo, que generalmente se manifestaban en forma de discapacidades de aprendizaje, de comportamiento, alteraciones de humor o enfermedades psicosomáticas". El estudio se basa en el análisis de tan solo diez niños de edades entre 18 meses y 14 años y mostraba algunas características comunes tales como un bajo nivel de autoestima y del concepto de sí mismos, habiendo experimentado situaciones de abandono y negligencia.

En el informe nacional británico se admite que existen ciertas pruebas de los problemas de tipo emocional, de comportamiento y de aprendizaje que expe- rimentan muchos hijos de consumidores de drogas problemáticas. Sin embargo, varios estudios realizados en el Reino Unido han mostrado que los hijos de padres drogadictos son comparables a los hijos de padres que no lo son y que tienen una situación social similar. Un estudio de 1996 concluyó que no había diferencia en el estado de salud y desarrollo de los niños nacidos y criados por madres que habían consumido sustancias opiáceas durante el embarazo y que continuaron con un tratamiento de metadona, al compararlos con un grupo de control de niños de edades y situación social similares ${ }^{16}$.

Las conclusiones globales de estos estudios europeos no justifican la necesidad de intervenciones específicas destinadas exclusivamente a los hijos de drogadictos. Los estudios austríacos, franceses, daneses y portugueses carecen del diseño conceptual necesario para probar la diferencia entre los hijos de los drogadictos y otros niños comparables de familias desfavorecidas (ningún grupo de comparación, pequeño número de muestreo) y no demostraron la especificidad de la influencia de la drogadicción paterna sobre los síntomas hallados. Por otra parte, los estudios irlandeses y británicos apoyan la necesidad de reforzar la cobertura psicosocial de los niños desfavorecidos en general, y no solamente de aquellos cuyos padres son drogadictos, según se expone más arriba.

Por lo tanto, las intervenciones en la primera infancia en el contexto del presente documento se refieren principalmente a actividades desarrolladas desde planteamientos "salutógenos" (Antonovsky7) y modelos de resistencia o fuerza moral que utilizan los recursos de la comunidad, de los padres y de los niños para desarrollar capacidades de prevención inespecíficas, en vez de centrarse exclusivamente en las capacidades de los padres drogadictos. Estas intervenciones pueden aplicarse a grupos destinatarios más amplios fuera del contexto terapéutico y hacer intervenir a una mayor diversidad de profesionales que no incluya sólo a los del sector dedicado a las toxicomanías. Esto no significa que no deban utilizarse los lugares de tratamiento para entrar en contacto con el subgrupo de drogadictos en los sectores vulnerables de la población con niños en situación de riesgo. No obstante, debe llamarse la atención sobre el hecho de que, un enfoque demasiado delimitado del tratamiento (la "patologización") no siempre resulta útil para desarrollar opciones equilibradas de salud pública para proteger las primeras fases de desarrollo de los niños. De la evidencia recogida, la ayuda a las familias de riesgo, independientemente de si se trata o no de drogadictos, es más beneficiosa para la prevención temprana en los niños y les supone una menor estigmatización.

Otro aspecto importante, que notoriamente se descuida en la prevención de la drogadicción, es la importancia de variables como el síndrome de déficit de atención (ADS - "attention deficit syndrome"). Es un 
elemento ampliamente conocido como factor de riesgo para el desarrollo posterior de toxicomanías, y un estudio longitudinal alemán ${ }^{18}$ muy reciente $(N=1252)$ ha identificado la hiperactividad/impulsividad (ADH) como variable predictiva importante, junto con el consumo temprano de cigarrillos, el hurto/vandalismo (en los hombres) y el bajo nivel de coherencia familiar, mientras que la toxicomanía parental tampoco en este estudio era un factor predictivo significativo. En la práctica, sin embargo, sabemos de pocas intervenciones dirigidas a este grupo infantil de riesgo, a pesar de la importancia de su incidencia actual en las sociedades occidentales.

Aunque la familia figure como la influencia primera y quizás la de mayor importancia en cómo una persona se enfrenta a las drogas más adelante en su vida, generalmente recibe una atención demasiado escasa en términos de medidas preventivas específicas y estructuradas. No cabe duda de que existe toda una plétora de intervenciones dirigidas a las familias, pero en su mayor parte su objetivo se limita a aumentar la concienciación de los padres sobre su papel en la prevención de la drogadicción. Sin embargo, la formación de capacidades y estilos concretos de educación parental desempeña un papel esencial para el desarrollo de factores protectores contra la toxicomanía incluso en la primera niñez, según sugieren los conocimientos actuales derivados de estudios científicos.

En los ámbitos de intervención ajenos a los servicios de tratamiento de la drogadicción, se plantea una cuestión logística importante: ¿cómo se llega a los niños en edad preescolar y a los grupos de riesgo? ¿Cómo llegar a las familias para concentrarse en las capacidades de educación parental? Para ello, la implicación asidua de los médicos de familia, pediatras y profesionales del cuidado de niños sería la estrategia más lógica y factible desde una perspectiva de sanidad pública, pero se utiliza muy raramente. La implicación de pediatras y profesionales del cuidado de niños en la prevención de la drogadicción podría ser crucial en las siguientes situaciones:

Durante y después del embarazo es posible entrar en contacto con ambos padres o con madres sin compañero para orientarles hacia las posibilidades existentes en cuanto a labores de prevención e implicarles en los programas. Podrá sensibilizarse a los padres sobre los efectos del uso prenatal de sustancias sobre los niños y sus efectos a largo plazo: tabaco, alcohol, y cannabis.

Los profesionales de la salud y del cuidado de niños ven pasar a un gran número de niños por sus servicios cada día. Tienen por lo tanto un papel importante que desempeñar en la identificación de aquellos niños que se encuentran en mayor situación de riesgo de desarrollar más adelante problemas relacionados con la droga: niños con signos del síndrome de déficit de atención, niños de los que se abusa o a los que se descuida, etc.... Adoptando estas estrategias es posible hacer que estos niños se beneficien de intervenciones con fines específicos.

Aconsejar y formar a los padres sobre la importancia de la prevención, los estilos de educación, relación y comunicación parentales. Los profesionales de la sanidad tienen un gran poder de persuasión sobre los padres y sobre los niños, y podrían desempeñar un papel primordial en la difusión de mensajes y técnicas de prevención.

El modo de comportamiento de los profesionales

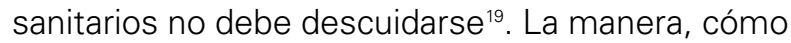
y para qué se recetan medicamentos (y entre ellos las sustancias psicoactivas), repercute en la conducta de padres e hijos con respecto a la salud, y podrá fomentarse el pensamiento crítico: ¿es preciso que todos los problemas (de salud, humor o comportamiento) se traten necesariamente mediante sustancias (recetadas)?

\section{NOTAS}

1 Künzel-Böhmer, J.; Bühringer, G.; Janik-Konecny, Th., "Expert Report on Primary Prevention of Substance Abuse" (Munich: IFT Institut für Therapieforschung, 1994), 78-80.

2 Hall, N.W.; Zigler, E., "Drug-Abuse Prevention Efforts for young children: A review and Critique of Existing Programs", American Journal of Orthopsychiatry vol. $67 \mathrm{n}^{\circ}$ 1 (enero de 1997): 124-143.

3 Wakschlag, LS., Hans, L.S., "Relation of maternal responsiveness during infancy to the development of behavious problems in high-risk youth". Developmental Psychology, vol. 35, n 2 (1999): 569-579.

4 Anda, R.F., Croft, J.B., Felitti, V.J. y otros, "Adverse Childhood Experiences and Smoking During Adolescence and Adulthood", JAMA vol. 282, n 17 (1999): 1652-8.

5 D'Apolito, K, "Substance Abuse: Infant and childhood outcomes", Journal of pediatric nursing vol. 13, n 5 (1998): 307-316.

6 Dubas, J.S.; Linche, K.B.; Galano, J. y otros, "Preliminary Evaluation of a resiliency-based preschool substance abuse and violence prevention project", Journal of Drug Education vol. 28, n³ (1998): 235-255.

7 Kämmerer, B., Expertise zur Sichtung vorhandener Materialien zur Suchtprävention im Vorschulalter (Colonia: Bundeszentrale für gesundheitliche Aufklärung, 1996).

8 Elling-de Boer, A., Invloed van ouders op het - en druggebruik van pubers. Literatuur research naar beschermende en risikofactoren in de opvoedin. Eerste resultaten, $9^{\circ}$ Forum Alcohol- en Drugs Onderzoek inglés (FADO), Utrecht, 4 de noviembre de 1999.

9 Hogan, D. M., The social and psychological needs of children of drug users: Report on exploratory study. (Dublín: The Children's Research Centre, 1997). 
10 Barnard, M., "Forbidden questions: drug-dependent parents and the welfare of their children". Addiction vol. $94 n^{\circ} 8$ (1999), 1109-1111.

11 Hogan, D. M., "Annotation: The psychological development and welfare of children of opiate and cocaine users: Review and research needs", Journal of Child Psychology and Psychiatri 39 (5) (1998): 609-620.

12 Gruber, C., Legl, T., Pernhaupt, G., Die Beziehung drogenabhängiger Mütter zu ihren Kindern Eine Kurzstudie anhand von Erfahrungen in der therapeutischen Arbeit in der Einrichtung Grüner Kreis (Manuscrito no publicado, sin fecha).

13 Olofsson M, Buckley W, Andersen GE, Friis-Hansen B. Investigación sobre 89 niños nacidos de madres drogodependientes, curso neonatal I. Acta Paediatrica Scandinavica (1983) 72: 403-6.

14 Bouchez J., Coppel A., Substitute treatment for the drug users. Ediciones Arnette 1997 ed. s.v., "Children of drug addicts: a need for special preventive treatment".
15 El Almeida, M. C. T. "Filho de peixe ... o medo e o mar: os filhos dos toxicodependentes ou o trabalho com crianças em risco", Toxicodependências, vol. $4 n^{\circ} 1$ (1998), 41-50.

16 Burns, E.C. y otros. "The health and development of children whose mothers are on methadone maintenance", Child Abuse Review, vol. 5. (1996) 113-122.

17 A. Antonovsky, Unraveling the Mystery of Heath (San Francisco: Jossey-Bass, 1987), 15-32.

18 Küfner, H., Duwe, A., Schuman, J., Bühringer, G., "Prädiktion des Drogenkonsums und der Suchtentwicklung durch Faktoren in der Kindheit: Grundlagen und Ergebnisse einer empirischen Studie, "Sucht vol. 46, $\mathrm{n}^{\circ} 1$ (2000) 32-53.

19 Reese, A., Konzepte der Suchtprävention im Kindes - und Jugendalter, Ärzteblatt Sachsen Anhalt 10 (1999) 18-22. 